\title{
A hybrid system: MnO-incorporated mesoporous silica nanoparticles for theranostic applications
}

\author{
VARUNA KUMARAVEL ${ }^{1}$, KARTIKEYAN DAMODARAN ${ }^{1}$, SAKTHIVEL GANDHI ${ }^{1,2}$ \\ and UMA MAHESWARI KRISHNAN ${ }^{1,2, *}$ \\ ${ }^{1}$ Centre for Nanotechnology and Advanced Biomaterials, SASTRA Deemed to be University, Thanjavur 613 401, India \\ ${ }^{2}$ Department of Chemistry, SASTRA Deemed to be University, Thanjavur 613 401, India \\ *Author for correspondence (umakrishnan@ sastra.edu)
}

MS received 10 May 2018; accepted 19 July 2018; published online 26 September 2018

\begin{abstract}
The need for an alternative $T_{1}$ contrast enhancer for magnetic resonance imaging (MRI) has been escalating owing to the toxicity profiles observed with the use of commercial contrast agents. Manganese oxide nanoparticles provide an optimal solution for the problem, as it is an endogenous co-factor for many enzymes in the biological system. In the present work, we have synthesized mesoporous silica nanoparticles encapsulated with manganese oxide nanoparticles as a positive contrast enhancer for MRI applications. Spherical magnetic MnO nanoparticles with divalent oxidation state were also synthesized and utilized as control to compare the efficiency of the nano-hybrid system. MRI showed higher contrast enhancement with the use of nano-hybrid and the relaxivity value for $T_{1}$-weighted imaging was calculated to be $2.6 \mathrm{mg} \mathrm{ml}^{-1} \mathrm{~s}^{-1}$. Also, the developed system was validated for its usefulness as a therapeutic system through adsorption studies. Therefore, the nano-hybrid has the potential to be a competent MRI contrast enhancer that could be used for theranostic applications.
\end{abstract}

Keywords. MRI; contrast agents; magnetic nanoparticles; mesoporous silica; theranostics.

\section{Introduction}

Molecular imaging techniques are on-demand for the diagnosis of intricate pathological conditions in clinical domain owing to their high precision and reliability in the elucidation of biological processes and monitoring of disease conditions. Techniques such as fluorescence-mediated tomography and photo-acoustic tomography are under development, while conventional techniques such as magnetic resonance imaging (MRI), X-ray computed tomography (CT), ultrasound, optical imaging (OI), single-photon emission CT (SPECT) and positron emission tomography (PET) have all been established as critical procedures for the analysis of highly complicated medical conditions [1].

Among all these techniques, MRI is considered to be a superior modality as it provides high spatial and temporal resolution at cellular and sub-cellular levels [2]. MRI involves the application of a magnetic field to align the water protons, wherein radio frequency is used for the relaxation of the protons back to their equilibrium. The acquired signal is then constructed into a 3D image by combining all the tomographical data and the results in the reconstituted image [3]. It is a non-invasive technique, which furnishes detailed anatomical information based on the relaxation time of water protons in whole tissue samples [4]. Soft tissue contrast without the use of radio-isotopes or ionizing radiations is yet another advantage of MRI. Though it is the most preferred imaging technique for the examination of cardiovascular and nervous system, the degree of sensitivity for image delineation between normal and malignant tissues is poor [5]. This is due to the fact that the long relaxation time of water protons confers only minimal difference between tissues. Therefore, to augment the image contrast efficiency, contrast agents (CAs) are being used.

CAs are generally used to enhance the sensitivity of images obtained through MRI by modulating the relaxation time of water protons in any biological sample. Two different classes of CAs exist based on their ability to enhance either brightness or darkness of the images. Positive CAs interfere with the transverse relaxation (spin-lattice relaxation) process to produce hyper-intense signals, whilst negative contrast enhancers display hypo-intense signals due to their influence on the spin-spin relaxation process [6]. Susceptibility artefacts and difficulty in delineating the pathogenic conditions are the major setbacks of $T_{2}$ CAs [7].

Sensitivity and biocompatibility are the pivotal characteristics of any CA that could be used for clinical diagnosis. Furthermore, colloidal stability and complete excretion from the body through renal system are the additional features expected from the material that is chosen as CA. $T_{1}$ CAs are highly favoured due to their ability to enhance the brightness of the magnetic resonance image, as it demarcates pathological conditions well, and their effortless excretion through the renal system. Gadolinium and its complexes, with high 
relaxivity values, were the best known $T_{1} \mathrm{CA}$ till the report on nephrogenic systemic fibrosis (NSF) in renal impaired patients caused by gadolinium-based CAs (GBCAs) was released. Moreover, lack of long blood circulation time due to the rapid clearance through the kidney is yet another concern, which diminishes the renal function leading to NSF [8]. Several reports have proved that GBCAs lead to various medical conditions such as gadolinium-associated plaques (sclerotic bodies) in kidney, gadolinium storage condition (GSC) in bones and brain, and gadolinium deposition disease, which elicits host-destructive immunological responses $[9,10]$. This property also reduces the time within which the 3D data can be acquired. Nominal tumour specificity of GBCAs is an additional drawback. As a result, the need for an equivalent alternative still persists.

Manganese has also been used as a $T_{1} \mathrm{CA}$ [11]. In fact, there is a form of MRI called manganese-enhanced MRI (MEMRI) where $\mathrm{Mn}^{2+}$ ions are directly infused for brain imaging due to their ability to enter the neurons via calcium channels (both are bivalent). The crucial application of MEMRI is mapping the cellular activity, which is limited by the tendency of $\mathrm{Mn}^{2+}$ ions to cause toxicity at high doses. In the cardiovascular system, it causes acute toxicity while chronic toxicity damages the cerebral or the hepatobiliary system. To overcome all these concerns, the oxidized form of manganese is introduced for imaging tumour and other medical conditions. Manganese oxide (MON) has been taken into consideration as a CA due to its physiological function as a co-factor for many enzymes and its minimal toxicity in the biological system [12]. Due to its biocompatibility under appropriate conditions, MONs have prolonged blood circulation time, which provides enough time for the scan to acquire images with high resolution.

Nanomaterials have garnered much attention as their properties and applications can be tuned by switching between their size, shape, dimensions and crystalline phases. Significant advantages of nanoparticulate system in biological applications include biostability, surface functionalization, which endows the property of tunable biodistribution, and the ability to specifically reach the target cells with the aid of a targeting ligand $[13,14]$. Since nanoparticles (NPs) enhance the efficiency of theranostics, a new arena that combines disease diagnosis and therapy, nanoparticulate systems of MONs have been developed for MRI and other applications such as drug delivery and multi-modal imaging. These systems have passive targeting properties and show enhanced contrast. Moreover, the large surface area to volume ratio endows them high drug loading capacity and targeting ability.

In the present work, we aim to develop a nano-hybrid system containing MON-incorporated mesoporous silica nanoparticles (MSNs) for theranostic applications of MRI and drug delivery. The system was prepared by the introduction of manganese ion precursors in to the mesopores of MSNs through a post-synthesis method. The functionality of the system was then established through MRI
Table 1. Experimental conditions for the optimization of reaction parameters.

\begin{tabular}{lcccc}
\hline Experiment & $\begin{array}{c}\text { Temperature } \\
\left({ }^{\circ} \mathrm{C}\right)\end{array}$ & $\begin{array}{c}\text { Precursor } \\
\text { molar ratio }\end{array}$ & $\begin{array}{c}\text { Surfactant } \\
(\mathrm{g})\end{array}$ & Method \\
\hline 1 & 80 & $1: 1$ & - & Ultrasonication \\
2 & 80 & $1: 1$ & 1 & Ultrasonication \\
3 & 80 & $1: 1$ & - & Hydrothermal \\
4 & 80 & $1: 1$ & 1 & Hydrothermal \\
5 & 80 & $1: 10$ & 1 & Hydrothermal \\
6 & 60 & $1: 1$ & 1 & Hydrothermal \\
7 & 100 & $1: 1$ & 1 & Hydrothermal \\
\hline
\end{tabular}

and drug adsorption studies. Cytotoxicity profile of the nano-hybrid was also analysed. Bare MONs were utilized for the comparison of functional properties and to optimize the reaction conditions for the preparation of MONs inside the mesopores.

\section{Materials and methods}

All chemicals were procured from Sigma Aldrich, USA. Distilled water was used for all experiments. The chemicals were used as received without any further purification.

\subsection{Synthesis of MONs}

MONs were prepared from hydrothermally synthesized manganese carbonate using literature protocol with slight modifications [15]. The reaction conditions were optimized for the preparation of uniform sized MONs. The conditions are presented in table 1. Initially, manganese chloride solution was prepared in distilled water; $1 \mathrm{~g}$ of sodium dodecyl sulphate was used as surfactant. Sodium bicarbonate solution in distilled water was then added to this solution and kept in static condition at $80^{\circ} \mathrm{C}$ for $1 \mathrm{~h}$. Then, the solution was filtered and the product was washed with distilled water. The dried product was calcined at $400^{\circ} \mathrm{C}$ for $4 \mathrm{~h}$ to obtain black coloured MONs.

\subsection{Synthesis of nano-hybrid}

A post-synthesis method was employed for the development of MON-incorporated MSNs. Optimized condition of MONs preparation was used for the synthesis of MONs inside MSNs. The procedure for the synthesis of MSNs, here MCM-41 was used, was taken from our previous report [16]. MCM-41 and manganese chloride were taken in the ratio of 1:2. Sodium bicarbonate solution was then added to this solution and kept in static condition at $80^{\circ} \mathrm{C}$ for $1 \mathrm{~h}$. The solution was then filtered and the product was washed with distilled water. The dried product was calcined at $500^{\circ} \mathrm{C}$ for $4 \mathrm{~h}$ to obtain the nano-hybrid. 


\subsection{Characterization of MONs and nano-hybrid}

The morphology of the MONs and nano-hybrid was observed by scanning electron microscopy (SEM) (JSM 6701F, JEOL, Japan). The samples were imaged after coating with gold. Transmission electron microscopic (TEM) images were obtained from samples dispersed in ethanol and deposited on a carbon-coated copper grid (JSM 2100F, JEOL, Japan).

$\mathrm{X}$-ray diffraction (XRD) study was performed to elucidate the crystalline nature of MONs and nano-hybrid (D8 focus, Bruker, Germany). Surface analysis (X-ray photoelectron spectroscopy (XPS)) was carried out to confirm the oxidation state of manganese ions in MONs (K-alpha, Thermo Fisher, USA).

Thermal profiling and thermo-gravimetric analysis (TGDTA, Q600, TA Instruments, USA) of the precursor were performed to optimize the temperature at which MONs are formed.

\subsection{Stability studies}

Stability studies were conducted to establish the media stability of the nano-hybrid; $50 \mathrm{mg}$ of the nano-hybrid was incubated in phosphate-buffered saline (PBS) containing 1\% foetal bovine serum (FBS) at $37^{\circ} \mathrm{C}$. Samples were then taken at specific time intervals $(3,6$ and $15 \mathrm{~h})$ and centrifuged at $8,000 \mathrm{rpm}$ for $5 \mathrm{~min}$. The pellets were then dried and SEM analysis was performed to visualize the morphology of the nano-hybrid.

\subsection{Phantom agar gel preparation for MRI}

Agar solution $(2.5 \%(\mathrm{w} / \mathrm{v}))$ was prepared by heating $750 \mathrm{mg}$ of agar in $30 \mathrm{ml}$ of phosphate buffer ( $\mathrm{pH} 7.4$ ) in a microwave oven for $2 \mathrm{~min}$. MONs and nano-hybrid dispersions were prepared in PBS with concentration ranging from 0.02 to $0.8 \mathrm{mg} \mathrm{ml}^{-1}$. Thereafter, $8.4 \mathrm{ml}$ of the sample dispersion was added to $1.6 \mathrm{ml}$ of pre-heated agar solution to prepare the phantom agar gels. The gels were vortexed immediately for the thorough mixing of the samples in the gel.

\subsection{Measurement of contrast enhancement properties using MRI}

A 1.5 T clinical scanner was used for MRI of the samples. Six different sample slices were taken for better precision. Repetition time (TR) was varied from 100 to $5000 \mathrm{~ms}$ for $T_{1}$-weighted imaging of the samples with a fixed echo time (TE) of $10 \mathrm{~ms}$.

Signal intensities of the obtained images were then calculated using ROI function in ImageJ software. $T_{1}$ values were noted using exponential curve fitting of signal intensity $v s$. corresponding time values (TR) plot. Relaxivity values of the sample were calculated as the slope from the relaxation rate vs. concentration plot.

\subsection{Cytotoxicity studies}

Cytotoxicity studies were performed with U937 and A549 cell lines to evaluate the toxicological properties of MONs, bare MCM-41 and nano-hybrid. Initially, the cells were cultured in RPMI (U937) and DMEM (A549) with 10\% FBS. The cells were seeded in 96-well plates (U937-1 $1 \times 10^{4}$ cells per well and A549-4 $410^{3}$ cells per well) and cultured for $24 \mathrm{~h}$ at $37^{\circ} \mathrm{C}$ with $5 \% \mathrm{CO}_{2}$. After $24 \mathrm{~h}$, media were discarded and fresh media were added to the wells; $10 \mu$ l of sample suspensions in PBS was added to each well with concentration varying from 10 to $500 \mu \mathrm{g} \mathrm{ml}^{-1}$. Cells were then incubated for 24 and $48 \mathrm{~h}$.

Cytotoxicity assay was carried out after adding $10 \mu \mathrm{l}$ of the MTS tetrazolium compound to each well and incubating for $3 \mathrm{~h}$. Absorbance values were taken at $490 \mathrm{~nm}$. Untreated cells were taken as control for the calculation of cell viability.

\subsection{Drug adsorption studies}

Adsorption studies were conducted to assess the drug adsorption capacity of the nano-hybrid using 5-flurouracil as a model drug. Drug adsorption efficiency of the nano-hybrid was compared only to that of free MCM-41 since bare MONs showed no adsorption at all.

Kinetic studies were carried out to study the rate of adsorption and other kinetic parameters that influence the adsorption process; $10 \mathrm{ml}$ of $0.05 \mathrm{mg} \mathrm{ml}^{-1}$ drug solution (drug in distilled water) was taken for all kinetic experiments. Adsorbent amount was varied as 5, 20 and $100 \mathrm{mg}$ in the ratio of 1:10, 1:40 and 1:200, respectively. The solution was centrifuged at 4,000 rpm for $3 \mathrm{~min}$ at every interval. Absorbance of the supernatant was measured at different time intervals ranging from 15 to $120 \mathrm{~min}$ at $273 \mathrm{~nm}$. Different kinetics such as pseudo-first-order, pseudo-second-order and Elovich models were studied.

Isotherm models were also studied to elucidate the mechanism of drug adsorption. Drug concentration was varied as $0.01,0.05,0.1$ and $0.5 \mathrm{mg} \mathrm{ml}^{-1}$ and $20 \mathrm{mg}$ of adsorbent was used for all experiments.

\section{Results and discussion}

\subsection{Characterization of MONs and nano-hybrid}

SEM analysis of bare MONs showed the influence of each parameter on the size and morphology of the NPs (figure 1). Influence of different preparation methods (ultrasonication and hydrothermal) on the size and formation of MONs was observed and it was concluded that hydrothermal synthesis produced more uniform sized NPs than ultrasonication method (table 1). This could be attributed to the static conditions maintained in the former method, which provided uniform energy distribution throughout the process. Optimal 

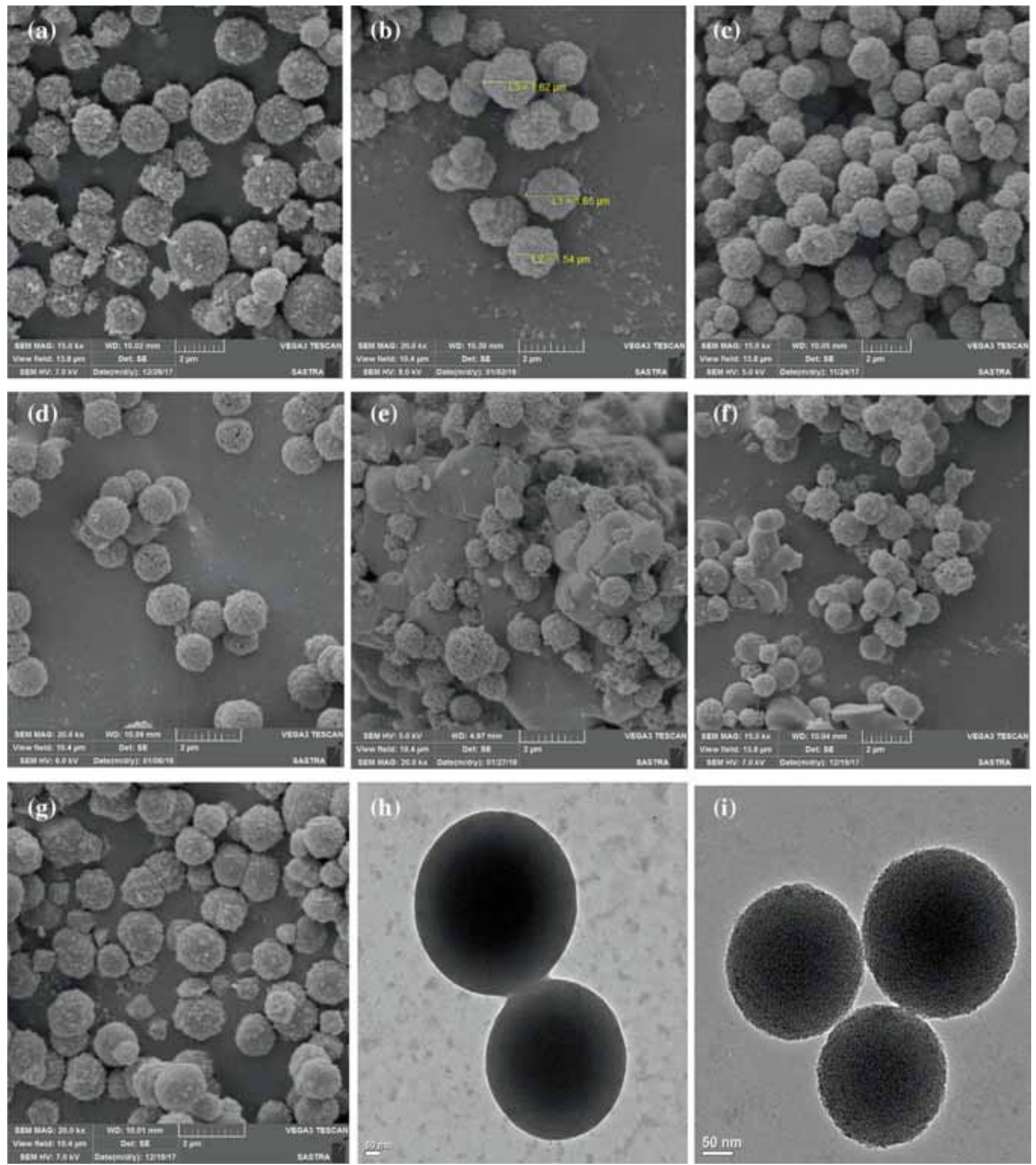

Figure 1. SEM images of MONs obtained through ultrasonication method: (a) without surfactant, (c) with surfactant and hydrothermal method, (b) without surfactant, (d) with surfactant, (e) at $60^{\circ} \mathrm{C}$, (f) at $100^{\circ} \mathrm{C}$ and (g) at precursor concentration ratio of $1: 10$. TEM image of (h) MONs synthesized under optimized condition and (i) nano-hybrid.

temperature for the reaction was found to be $80^{\circ} \mathrm{C}$ as the NPs prepared at 60 and $100^{\circ} \mathrm{C}$ resulted in non-uniform and distorted surface, respectively. Precursor ratio also plays a crucial role in the regulation of size of the NPs. Precursor ratio of 1:1 was found to produce monodisperse NPs when compared with those prepared at the ratio of 1:10, which proves that an optimal interaction between the precursors occurred when they were used at the same concentration 

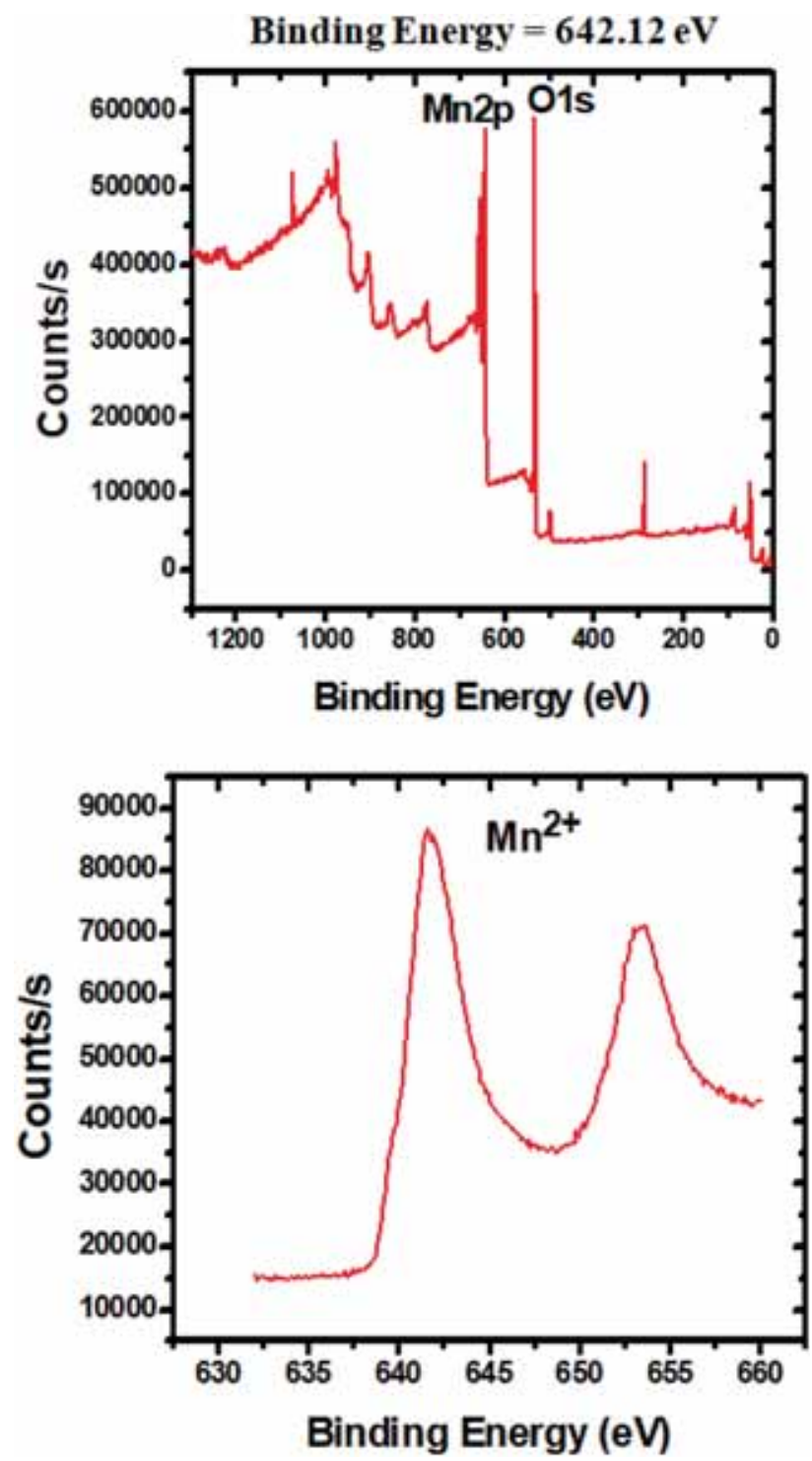

Figure 2. XPS scan of MONs with Mn elemental scan.

rather than at varying concentrations. Effect of surfactant was studied as it can lead to the formation of smaller particles by confining the crystal growth of the NPs [17]. The same phenomenon was observed in our study, where the NPs obtained with the use of surfactant showed a decrease in the overall particle size with uniformity in particle morphology. The use of surfactant was studied only for the synthesis of bare MONs as the nano-hybrid was formed using MSNs as the template for the formation of MONs inside the mesopores. SEM and TEM analyses of NPs synthesized at optimized condition showed MONs with an average particle size of $800 \mathrm{~nm}$. These results showed that process conditions are highly vital for the regulation of the overall dimension of the MONs and thus the optimized variables were used for the synthesis of MONs using MSNs as the template.

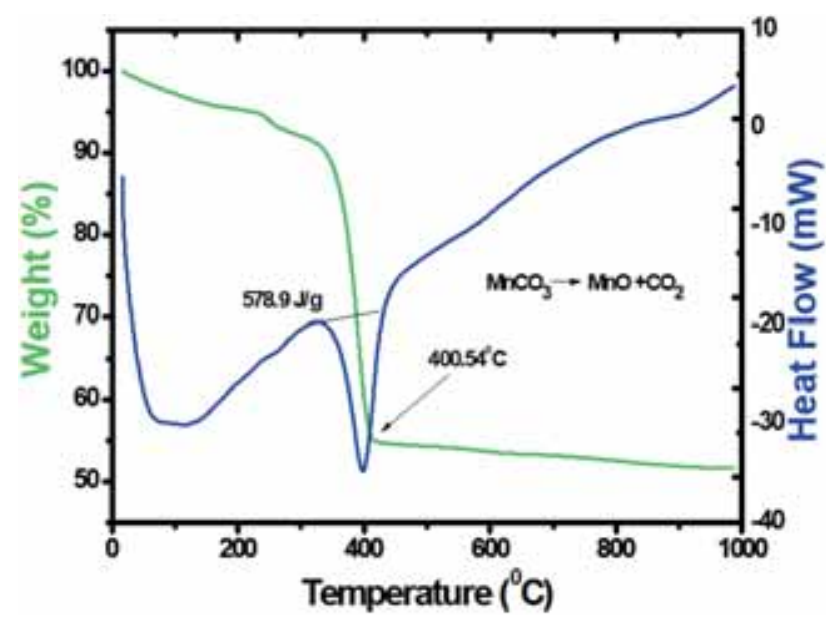

Figure 3. Simultaneous thermal analysis of $\mathrm{MnCO}_{3}$.

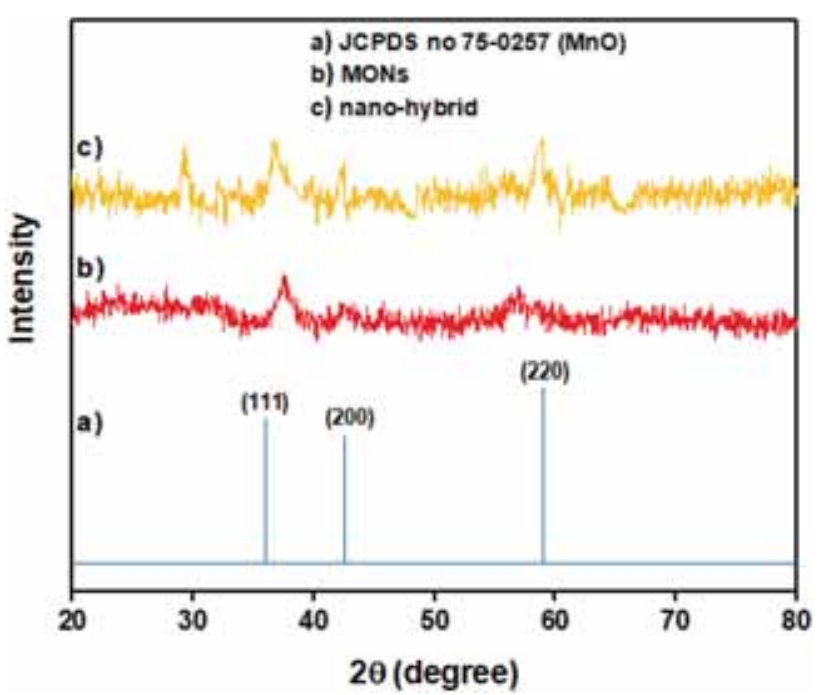

Figure 4. XRD patterns of MONs and nano-hybrid.

Simultaneous thermal analysis (STA) of the precursor $\left(\mathrm{MnCO}_{3}\right)$ was carried out to observe the reaction progress at different temperatures. It was found that the thermal decomposition of $\mathrm{MnCO}_{3}$ to $\mathrm{MnO}$ with the release of $\mathrm{CO}_{2}$ occurred at $400^{\circ} \mathrm{C}$, which was confirmed by the $40.09 \%$ weight loss of the sample at the same temperature. This further validated the formation of $\mathrm{MnO}$ with oxidation state $2+$. The observed result is in accordance with the theoretical calculations reported for \% weight loss in literature [18].

$$
\mathrm{MnCO}_{3}(\mathrm{~s}) \longrightarrow \mathrm{MnO}(\mathrm{s})+\mathrm{CO}_{2}(\mathrm{~g})
$$

Oxidation state of the MONs was further confirmed through XPS analysis. The characteristic peak at $642.12 \mathrm{eV}$ corresponds to the Mn 2p state, which is ascribed to the $\mathrm{Mn}^{2+}$ valence state of MONs. This is a much needed substantiation 

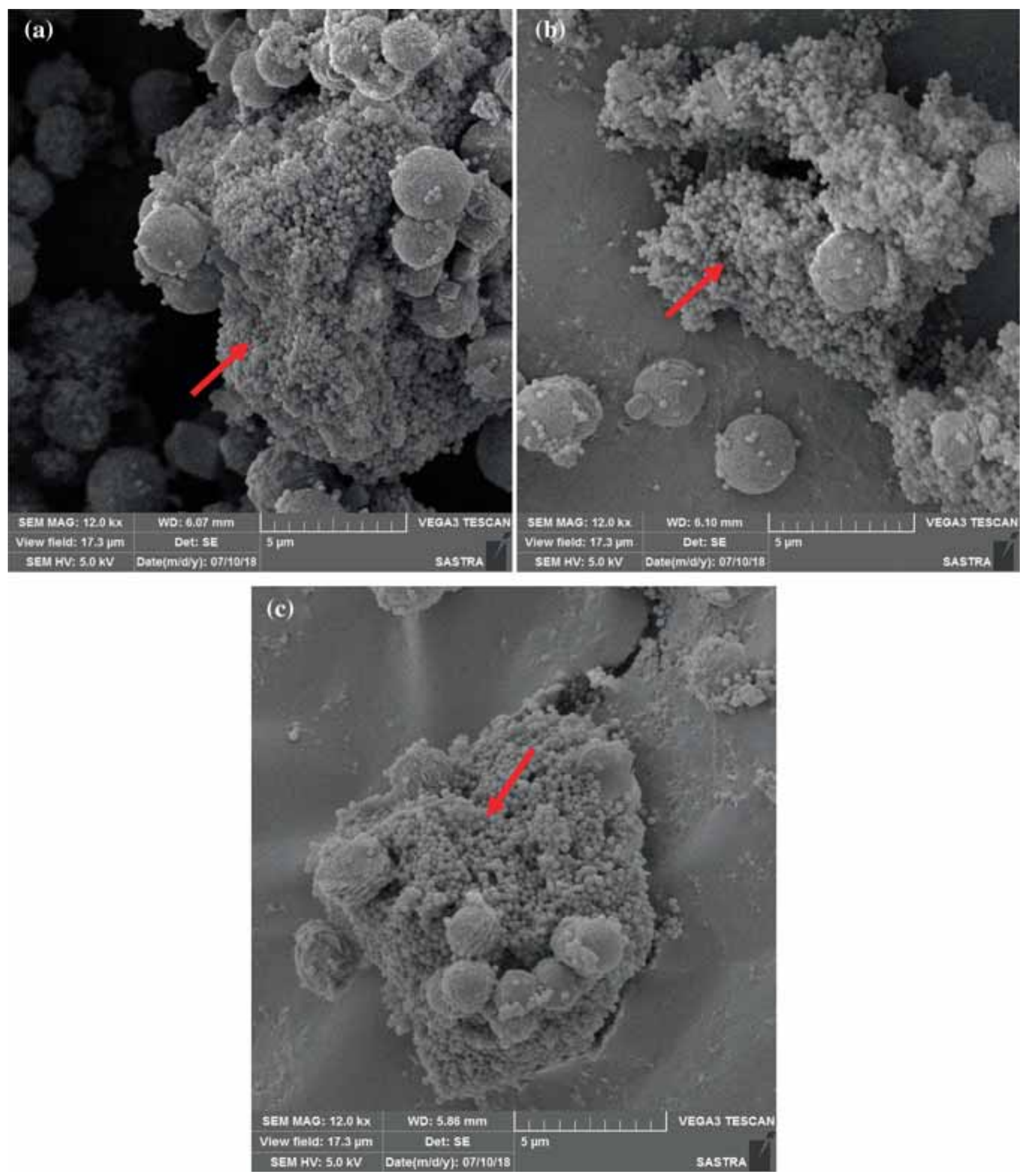

Figure 5. SEM images of the nano-hybrid incubated in PBS containing $1 \%$ FBS for (a) 3, (b) 6 and (c) $15 \mathrm{~h}$ (the red arrows indicate the nano-hybrid).

for the chemical state of MONs, as the contrast enhancement property of the NPs is reported to diminish with the increase in the oxidation state or in other words, with decrease in the number of unpaired electrons in the valence shell [19].
The peak at $530.13 \mathrm{eV}$ is attributed to the metal-oxygen binding. Peak splitting of Mn $2 \mathrm{p}$ was observed at $653.68 \mathrm{eV}$ (Mn 2p1/2) and $641.68 \mathrm{eV}$ (Mn 2p3/2) [20]. All these observations validate the $\mathrm{Mn}^{2+}$ chemical nature of the MONs 


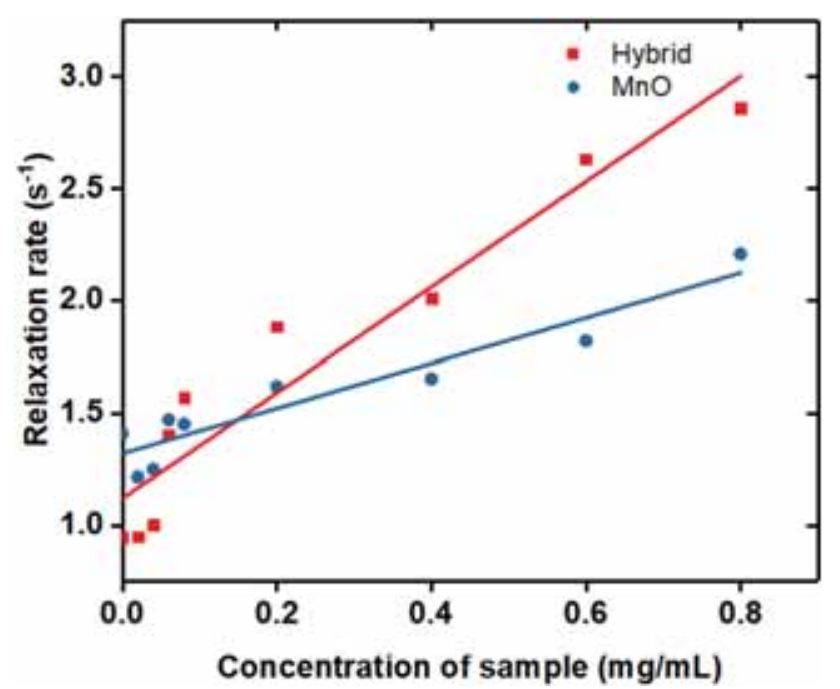

Figure 6. $T_{1}$ relaxation plot showing the relaxation rate of the MONs and nano-hybrid. synthesized under optimal conditions. Thus, the synthesized MONs was observed to be $\mathrm{MnO}$ with the oxidation state of $2+$, which is desirable for contrast enhancement properties (figures 2 and 3 ). The same experimental conditions were used for the synthesis of MONs inside the mesopores of MSNs.

TEM analysis was performed to elucidate the structural features of the nano-hybrid and to observe the MONs in the mesopores. Results confirmed the presence of MONs inside the mesopores, i.e., electron-dense regions in the TEM image (since it is a metal oxide) [21]. Furthermore, the average particle size was found to be $200 \mathrm{~nm}$.

The crystalline nature of the MONs and nano-hybrid was studied by XRD (figure 4). Experimental results confirmed the presence of cubic crystal structure in both MONs and nano-hybrid (JCPDS no 75-0257). Broadening of the diffraction peaks could be attributed to the nanocrystalline nature of MONs and nano-hybrid [22]. The successful synthesis of MONs inside the mesopores was validated by the
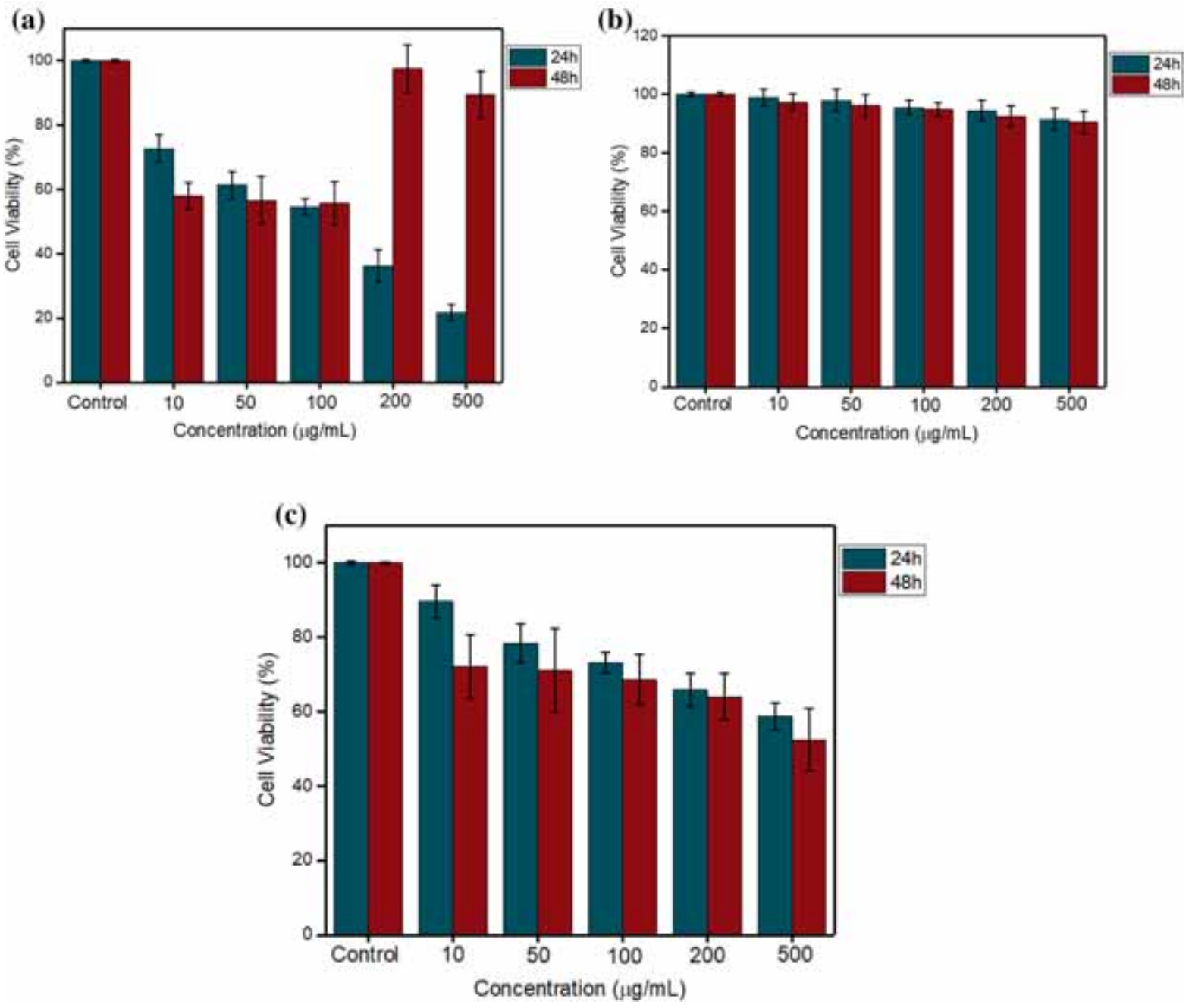

Figure 7. Cytotoxicity data using U937 cell lines for (a) MONs, (b) MCM-41 and (c) nano-hybrid. 
(a)

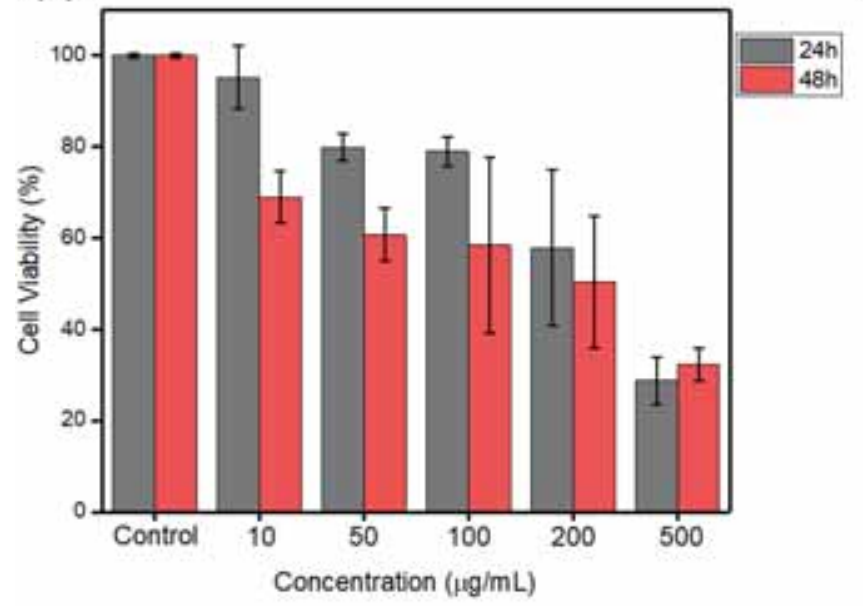

(b)

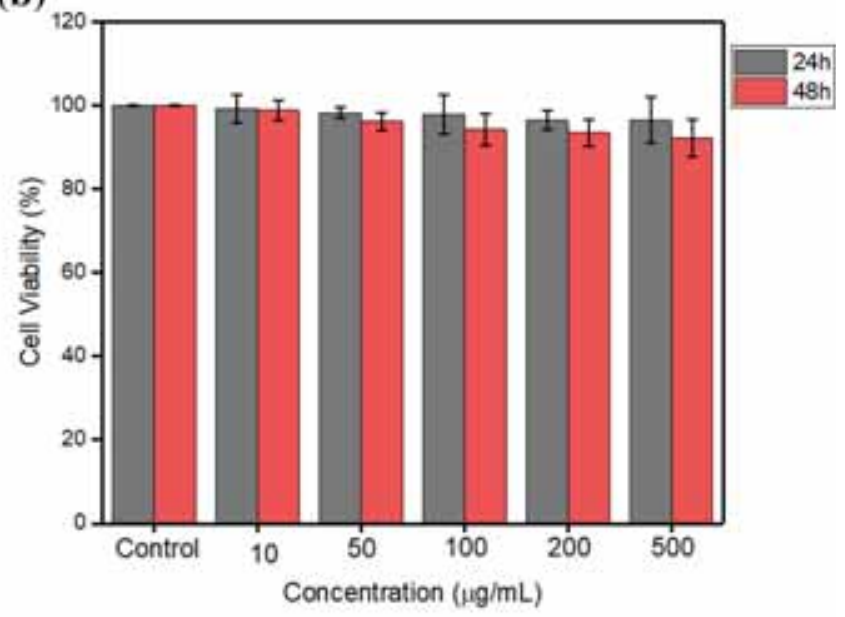

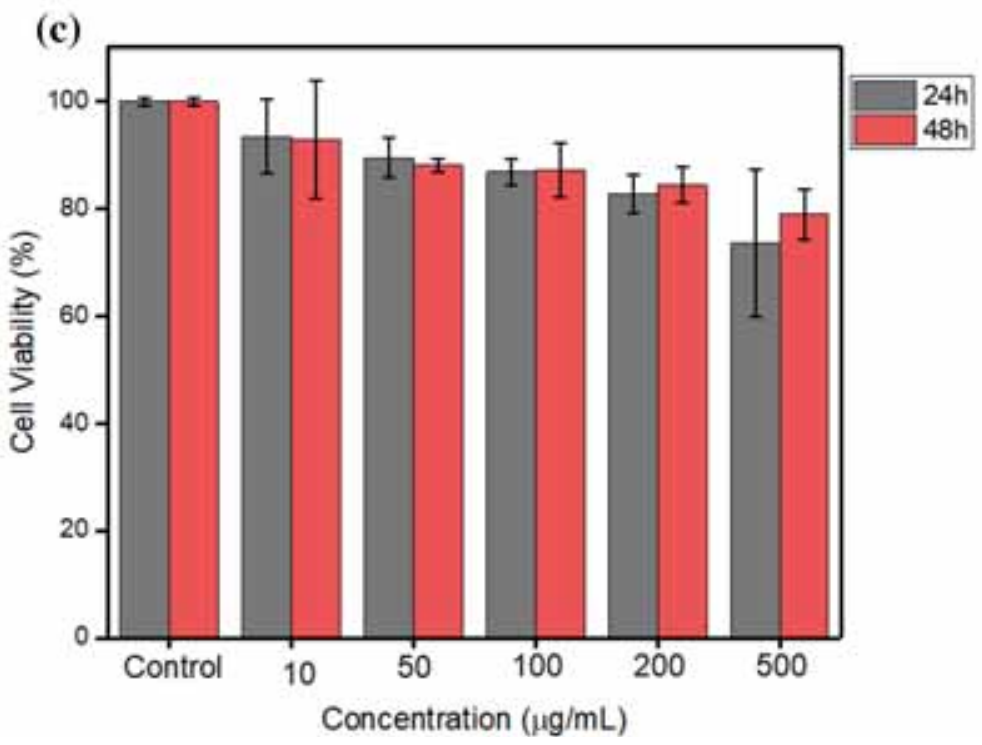

Figure 8. Cytotoxicity data using A549 cell lines for (a) MONs, (b) MCM-41 and (c) nano-hybrid.

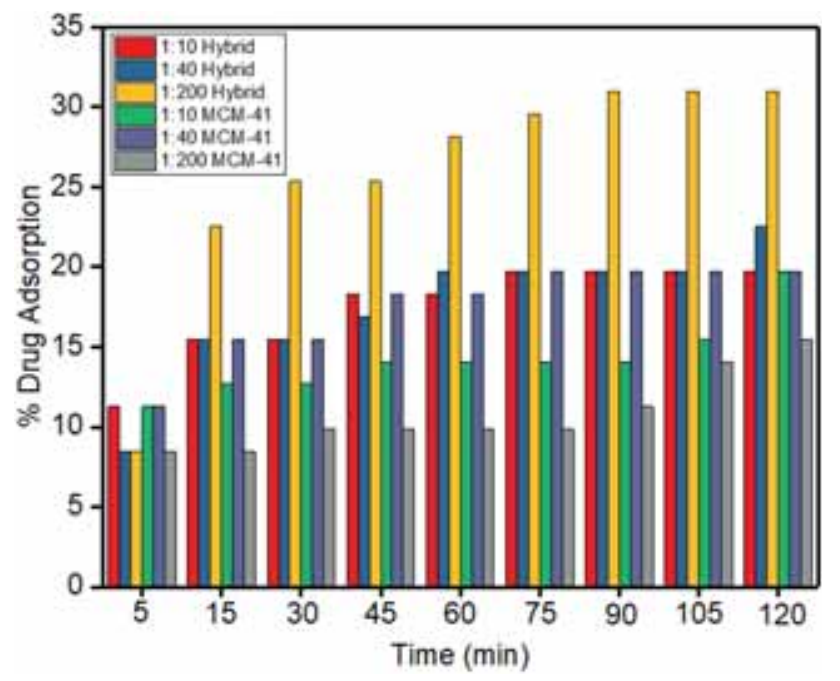

Figure 9. Drug adsorption data for nano-hybrid and MCM-41. presence of diffraction peak around $29^{\circ}$, since it indicates the presence of amorphous silica in the nano-hybrid [23].

SEM analysis of the nano-hybrid, which was incubated in PBS containing $1 \%$ FBS, was performed to characterize the medium stability of the developed hybrid (figure 5). As mentioned earlier, the particle size of the nano-hybrid was around $200 \mathrm{~nm}$. The same particle size was maintained even after an incubation period of $15 \mathrm{~h}$ in the medium, which validated the ability of the nano-hybrid to perform well in the medium without aggregation. Thus, the experimental data provided an evidence for the potential of the nano-hybrid to be stable under culture media conditions [24].

\section{$3.2 M R I$}

MRI of MONs and nano-hybrid was carried out to compare the potential between $\mathrm{MnO}$ and nano-hybrid to be used for contrast enhancement (figure 6). Signal intensities of 

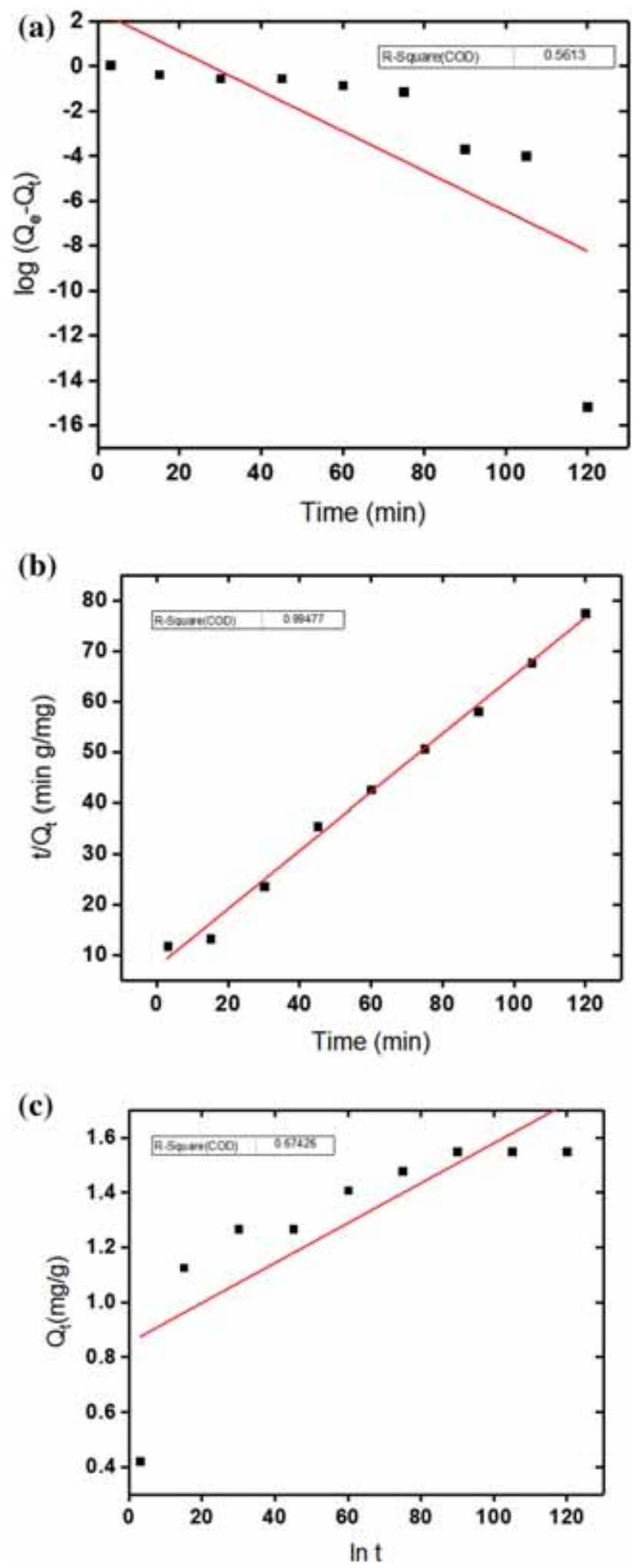

Figure 10. Kinetic modelling of MCM-41: (a) pseudo-first order, (b) pseudo-second order and (c) Elovich model.

the obtained images were higher with the use of nano-hybrid rather than the MONs. This could be explained through the mechanism of spin-lattice relaxation.
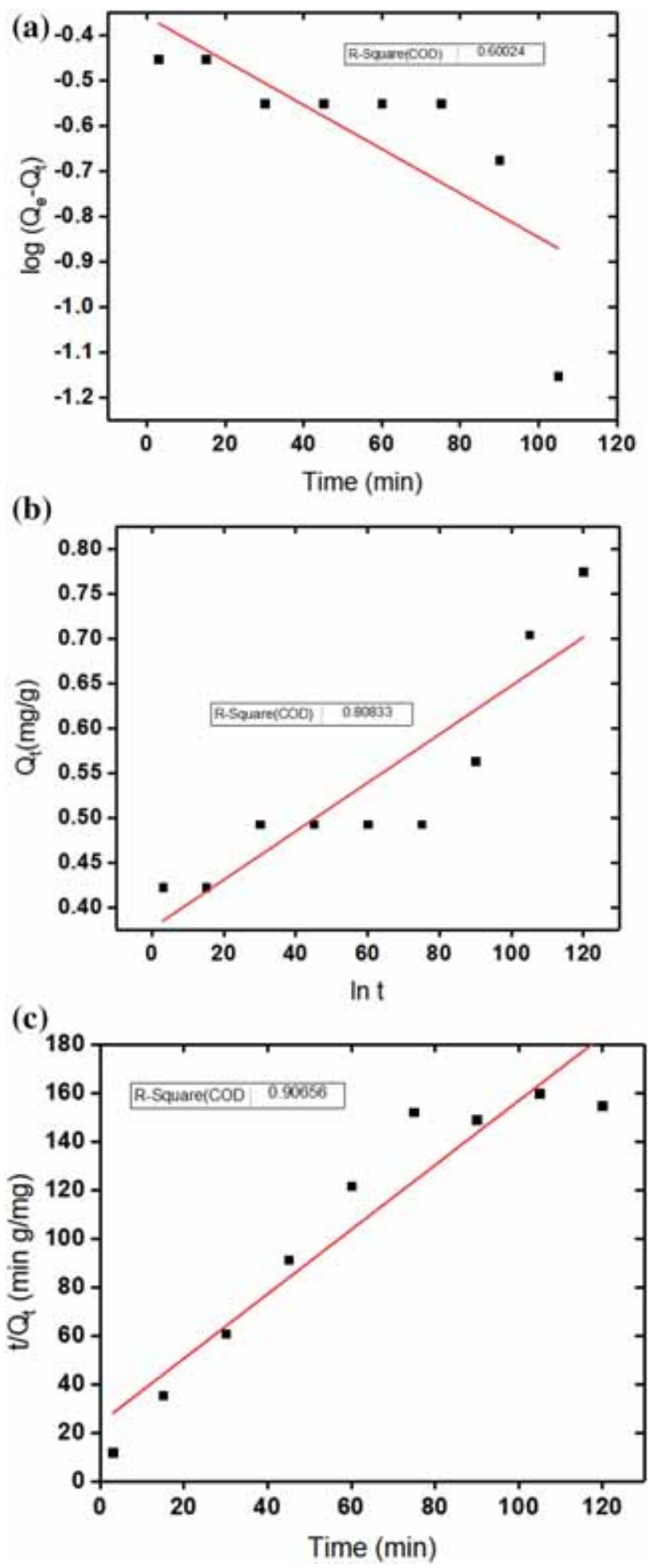

Figure 11. Kinetic modelling of nano-hybrid: (a) pseudo-first order, (b) pseudo-second order and (c) Elovich model.

$T_{1}$ relaxation occurs as a result of energy dissipation to the surrounding environment from the spins. The crucial role of CA is to elevate the energy transfer through its interaction with the water protons. Since the developed system contains MONs 

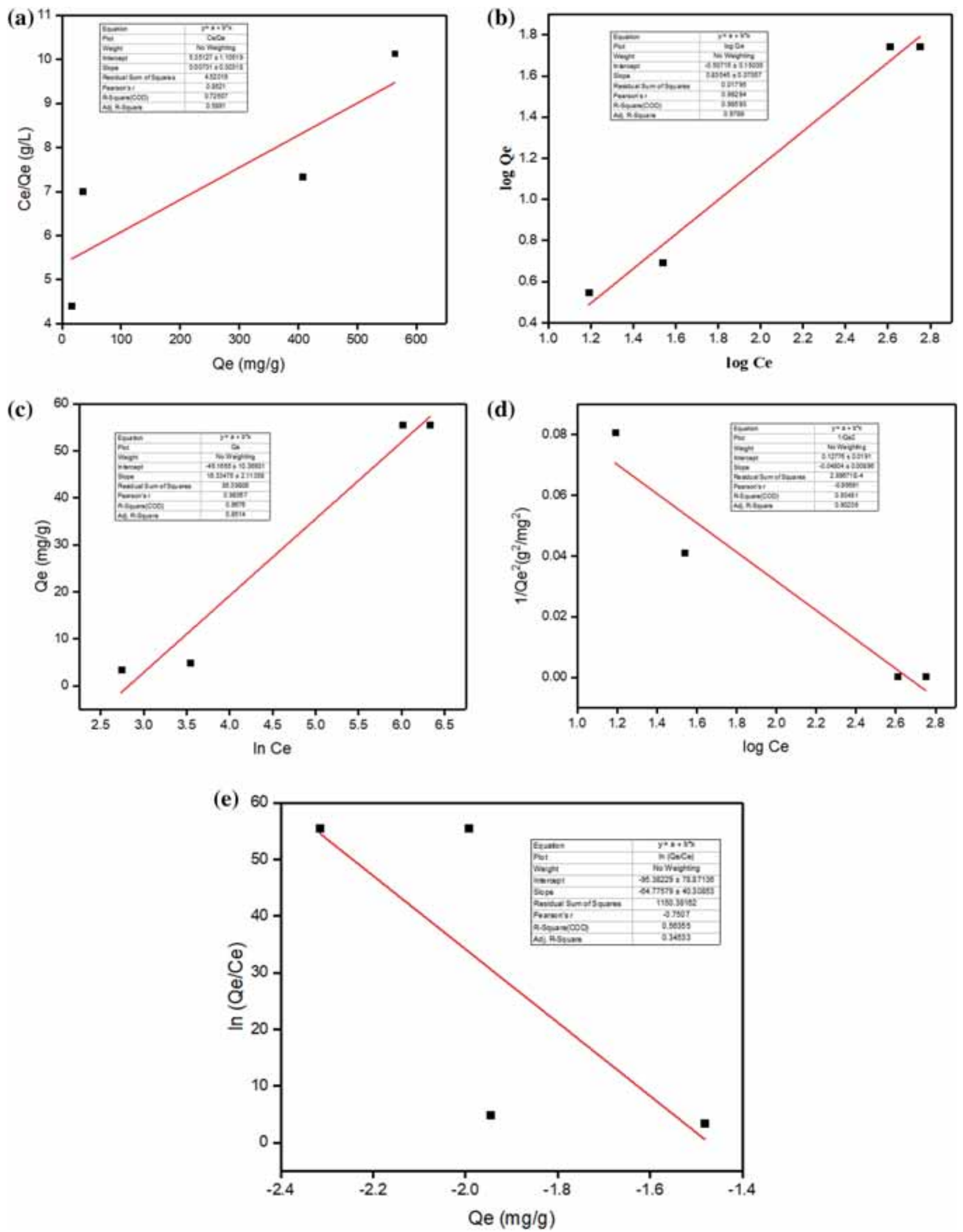

Figure 12. Adsorption isotherm modelling of MCM-41: (a) Langmuir model, (b) Freundlich model, (c) Temkin model, (d) Harkins-Jura model and (e) Elovich model. 

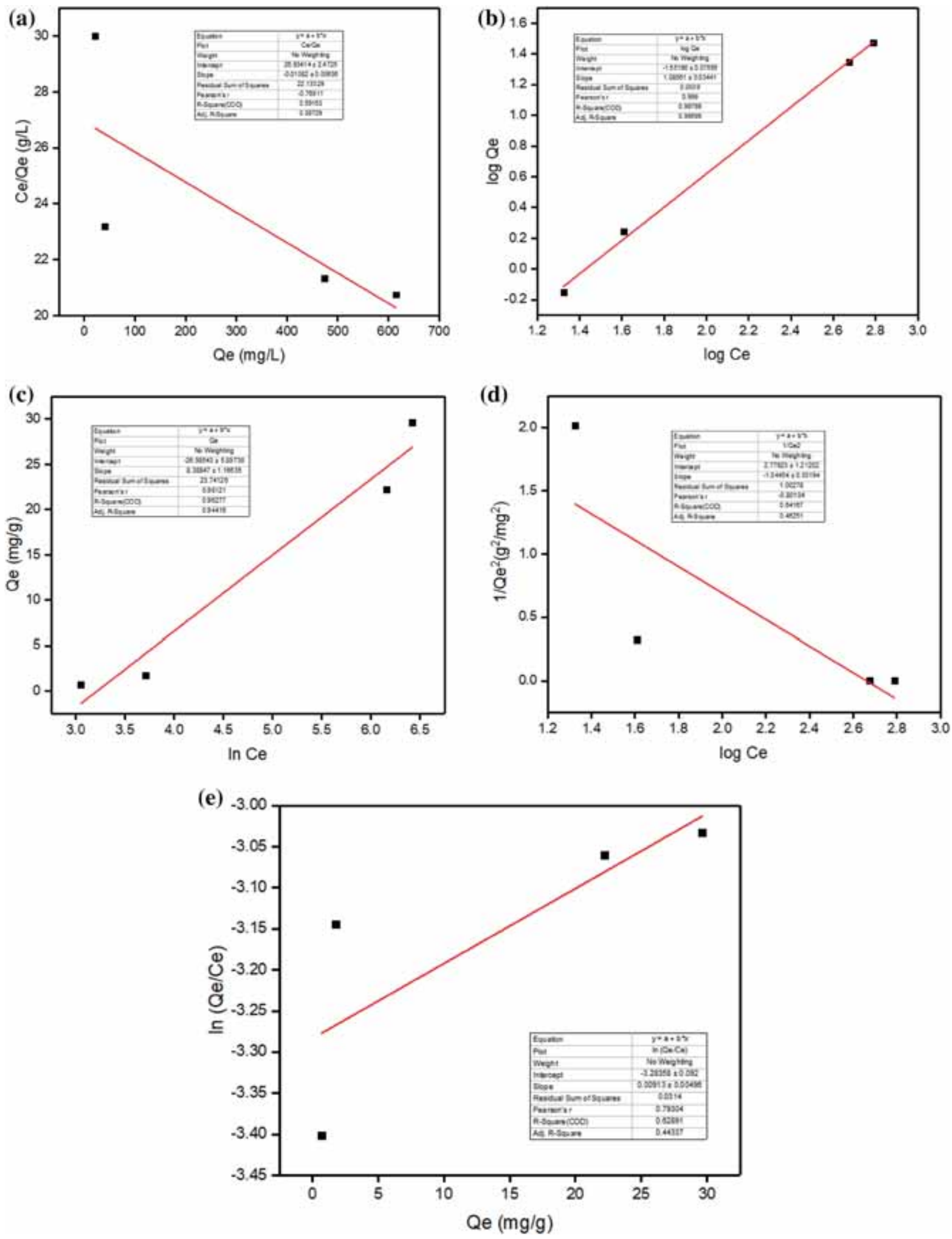

Figure 13. Adsorption isotherm modelling of nano-hybrid: (a) Langmuir model, (b) Freundlich model, (c) Temkin model, (d) Harkins-Jura model and (e) Elovich model. 
inside MSNs, the tumbling motion of the NPs is reduced and confined. According to the SBM theory, correlation time of the NPs is the key variable that governs its relaxivity. Thus, the presence of mesopores (a) allows the anisotropic diffusion of water molecules towards the magnetic core, which is the fastest diffusion occurring along the channels [25], (b) provides a confined environment wherein the motion of the NP is restricted, thereby increasing the interaction between the NPs and water molecules, which is in accordance with the SBM theory [26] and also (c) enhances the inner sphere effect, where the contrast enhancement is due to the interaction between the electrons of the magnetic material and the protons present in the first hydration shell [27]. Furthermore, an increase in the signal intensity with respect to increase in concentration was also observed as reported before [28].

Relaxivity value of the nano-hybrid was calculated to be $2.6 \mathrm{mg} \mathrm{ml}^{-1} \mathrm{~s}^{-1}$, which is relatively similar to that of the commercially available contrast enhancement systems, whilst for MONs, it is $1 \mathrm{mg} \mathrm{ml}^{-1} \mathrm{~s}^{-1}$. Thus, the nano-hybrid developed from hydrothermally synthesized $\mathrm{MnCO}_{3}$ has been confirmed to be a promising $T_{1}$ contrast enhancement agent for MRI.

\subsection{In vitro toxicity studies}

MTS assay was performed to estimate the cytotoxicity of the nano-hybrid and MONs (figures 7 and 8). It was observed that higher percentage of cells were viable (70-90\% of both the cell lines) with the use of nano-hybrid, whilst the cell viability was found to be only $25 \%$ (at higher concentration) at $48 \mathrm{~h}$ for both the cell lines with the administration of bare MONs. Mild cytotoxicity at high doses could be attributed to the acidic conditions present in cells, which influence the leaching of $\mathrm{Mn}$ ions [29]. These results confirm the relative non-cytotoxic nature of the nano-hybrid even at higher doses $\left(500 \mu \mathrm{g} \mathrm{ml}^{-1}\right)$ [30]. Microscopic visualization of the cells revealed that most of the administered MONs were retained outside the cells at $48 \mathrm{~h}$ on dosing with higher concentrations. This explained the higher viability percentage observed even at higher doses after incubation for longer period of time. Cytotoxic nature of MCM-41 was also studied and the results established the non-cytotoxic nature of MCM-41, as the cell viability was almost $90 \%$ at higher doses even after $48 \mathrm{~h}$ of incubation for both the cell lines.

\subsection{Drug adsorption studies}

Drug adsorption studies were performed to establish the potential of the nano-hybrid to act as a drug carrier (figure 9). No adsorption was observed with the use of bare MONs as the adsorbent. This could to be attributed to the non-porous nature of bare MONs. Experimental data showed that the maximum drug adsorption was $30 \%$ for nano-hybrid and $15 \%$ for MCM-41 at higher drug to material ratio. Adsorption of the drug could be attributed to the large surface area to volume ratio of the nano-hybrid and the presence of pores.

Adsorption is a phenomenon wherein the interaction between the adsorbent and the adsorbate occurs in three distinct steps: (1) external diffusion of the adsorbate from the bulk solution to the adsorbent surface, (2) internal diffusion of the adsorbate to the adsorption sites and (3) the adsorption. Kinetic models are based on the assumption that the rate-limiting step is adsorption rather than diffusion [31]. Best fits of the kinetic models were selected based on the correlation coefficient values (figures 10 and 11). It was found that the adsorption system obeyed pseudo-second-order kinetics, which explains the adsorption process in terms of first-order rate equation. That is, the adsorption process is governed by the concentration of both the reactants (drug and the material). This also shows that there is no interaction between the drug molecules [32]. It was also observed that the experimental drug adsorption capacity values $\left(Q_{\mathrm{e}}=\right.$ $0.77 \mathrm{mg} \mathrm{g}^{-1}$ ) were highly correlated to the theoretical values $\left(Q_{\mathrm{e}}=0.75 \mathrm{mg} \mathrm{g}^{-1}\right)$ obtained from the kinetic plot of nano-hybrid.

Different isotherm models such as Langmuir, Freundlich, Temkin, Harkin-Jura and Elovich were examined to study the nature of adsorption (figures 12 and 13). The nano-hybrid was observed to follow the Freundlich isotherm model, which proposes the heterogeneity of the process. This means that the adsorption sites are distributed heterogeneously throughout the adsorbent surface [33]. This could be ascribed to the presence of pores on the surface of the nano-hybrid. These reports show that the nano-hybrid could be used for drug delivery applications.

\section{Conclusion}

Herein, we have developed a novel nano-hybrid with MONs encapsulated in the mesopores of MSNs through postsynthesis method. The efficiency of the nano-hybrid has been attributed to the confinement of the magnetic material by the pores. The experimental results established the ability of the nano-hybrid to be applied as a CA for $T_{1}$-weighted MRI. Since the pore volume could further accommodate guest molecules, the material could also be applied for drug delivery and this was tested with adsorption studies using a model drug. The results showed that the nano-hybrid has the potential for therapeutic applications. Cytotoxicity studies revealed the near-non-toxic nature of the hybrid. Further studies on the biodistribution would provide an insight into the thorough functionality of the nano-hybrid as a theranostic system.

\section{Acknowledgements}

We wish to acknowledge Nano Mission Council, DST (DST/INT/JSPS/P-221/2016) and SASTRA Deemed University for the financial and infrastructural support. 


\section{References}

[1] Berger A 2002 Br. Med. J. 32435

[2] Chaughule R S, Purushotham S and Ramanujan R V 2012 Proc. Natl. Acad. Sci. 82257

[3] Estelrich J, Sánchez-Martín M J and Busquets M A 2015 Int. J. Nanomed. 101727

[4] Gao H, Liu X, Tang W, Niu D, Zhou B, Zhang H et al 2016 Nanoscale 819573

[5] Gao Z, Ma T, Zhao E, Docter D, Yang W, Stauber R H et al 2016 Small 12556

[6] Guillet-Nicolas R, Laprise-Pelletier M, Nair M M, Chevallier P, Lagueux J, Gossuin Y et al 2013 Nanoscale 511499

[7] Kim J, Piao Y and Hyeon T 2009 Chem. Soc. Rev. 38 372

[8] Lee S H, Kim B H, Na H B and Hyeon T 2014 Wiley Interdiscip. Rev. Nanomed. Nanobiotechnol. 6196

[9] Liu X, Chen C, Zhao Y and Jia B 2013 J. Nanomater. 20131

[10] Ramalho J, Ramalho M, Jay M, Burke L M and Semelka R C 2016 Magn. Reson. Imaging 341394

[11] Semelka R C, Ramalho M and Jay M 2016 Magn. Reson. Imaging 341399

[12] Wang A, Guo M, Wang N, Zhao J, Qi W, Muhammad F et al 2014 Nanoscale 65270

[13] Wang P, Yang J, Zhou B, Hu Y, Xing L, Xu F et al 2016 ACS Appl. Mater. Interfaces 947

[14] Xu W, Kattel K, Park J Y, Chang Y, Kim T J and Lee G H 2012 Chem. Phys. 1412687

[15] Pourmortazavi S M, Rahimi-Nasrabadi M, DavoudiDehaghani A A, Javidan A, Zahedi M M and Hajimirsadeghi S S 2012 Mater. Res. Bull. 471045
[16] Murugan B, Ramana L N, Gandhi S, Sethuraman S and Krishnan U M 2013 J. Mater. Chem. B 13494

[17] Park M, Lee N, Choi S H, An K, Yu S H, Kim J H et al 2011 Chem. Mater. 233318

[18] Pinc J, Jankovský O and Bartůněk V 2017 Chem. Pap. 711031

[19] Xiao J, Tian X M, Yang C, Liu P, Luo N Q, Liang Y et al 2013 Sci. Rep. 33424

[20] Li J, Wu C, Hou P, Zhang M and Xu K 2018 Biosens. Bioelectron. 1021

[21] Schladt T D, Koll K, Prüfer S, Bauer H, Natalio F, Dumele O et al 2012 J. Mater. Chem. 229253

[22] Chen N, Shao C, Qu Y, Li S, Gu W, Zheng T et al 2014 ACS Appl. Mater. Interfaces 2219850

[23] Yang H, Zhuang Y, Hu H, Du X, Zhang C, Shi X et al 2010 Adv. Funct. Mater. 111733

[24] Chen F, Zhao Y, Pan Y, Xue X, Zhang X, Kumar A et al 2015 Mol. Pharm. 122237

[25] Kim T, Momin E, Choi J, Yuan K, Zaidi H, Kim J et al 2011 J. Am. Chem. Soc. 1332955

[26] Gandhi S, Venkatesh S, Sharma U, Jagannathan N R, Sethuraman S and Krishnan U M 2011 J. Mater. Chem. 211569

[27] Hsu B Y, Ng M, Zhang Y, Wong S Y, Bhakoo K, Li X et al 2015 Adv. Funct. Mater. 255269

[28] Shin J, Anisur R M, Ko M K, Im G H, Lee J H and Lee I S 2009 Angew. Chem. Int. Ed. 48321

[29] Niu D, Luo X, Li Y, Liu X, Wang X and Shi J 2013 ACS Appl. Mater. Interfaces 59942

[30] Hu H, Dai A, Sun J, Li X, Gao F, Wu L et al 2013 Nanoscale 510447

[31] Largitte L and Pasquier R 2016 Chem. Eng. Res. Des. 109495

[32] Ho Y S and McKay G 2002 Adsorp. Sci. Technol. 20797

[33] Ayawei N, Ebelegi A N and Wankasi D 2017 J. Chem. 20171 